abatacept therapy. Proportions of CD14 monocytes and phenotypic characteristics, including the expression of major histocompatibility complex class II molecules, co-stimulatory molecules and adhesion molecules on CD14 monocytes, were determined by flow cytometry. In addition, PBMCs were incubated with different concentrations of abatacept overnight and CD14 monocytes were analysed for the expression of adhesion molecules by flow cytometry. Finally, isolated monocytes were analysed for their migratory behaviour using an in vitro model of transmigration through an endothelial cell barrier.

Results The authors observed a substantial increase in the proportions of CD14 monocytes in patients treated with abatacept. Comparative phenotypic analyses of CD14 monocytes before and after the initiation of abatacept therapy revealed no differences in the expression of co-stimulatory molecules. On the other hand, adhesion molecules such as CD15 and CD54 were downregulated. In addition, a dose-dependent downregulation of CD15, CD54 and CD102 expression was observed after $24 \mathrm{~h}$ of in vitro culture in the presence of abatacept. In line with these phenotypic changes, isolated monocytes displayed a significantly reduced migratory capacity upon abatacept therapy.

Conclusion Until now our data suggest that abatacept not only influences effector $\mathrm{T}$ cell populations in RA but also changes proportions and functional properties of peripheral blood monocytes. Abatacept may therefore interfere with the migration of monocytes into the synovial tissue of the joint which might contribute to the beneficial effects of abatacept treatment in patients with RA.

\title{
A169 EFFECTS OF ABATACEPT ON MONOCYTES IN PATIENTS WITH RHEUMATOID ARTHRITIS
}

M Bonelli, E Ferner, A Savitskaya, S Blüml, C-W Steiner, E Rath, J S Smolen, C Scheinecker Medical University of Vienna, Austria

10.1136/ard.2010.129668f

Introduction Abatacept represents a soluble recombinant human fusion protein comprising the extracellular domain of human CTLA-4 and a fragment of the modified Fc domain of human IgG1 (CTLA-4Ig). Due to the interaction with CD80/ CD86, abatacept inhibits the binding of CD28 and thereby effector $\mathrm{T}$ cell activation. In addition, inhibition of CD80/ CD86 binding might also exert effects on antigen presenting cells including cells of the myelo-monocytic lineage. The authors therefore performed phenotypic and functional analysis of monocytes in patients with rheumatoid arthritis (RA) before and after the initiation of abatacept therapy.

Methods Peripheral blood mononuclear cells (PBMCs) were collected from RA patients before and after the initiation of 